\title{
Existing data sources for clinical epidemiology: The Danish National Database of Reimbursed Prescriptions
}

This article was published in the following Dove Press journal:

Clinical Epidemiology

10 November 2012

Number of times this article has been viewed

\section{Sigrun Alba Johannesdottir Erzsébet Horváth-Puhó \\ Vera Ehrenstein \\ Morten Schmidt \\ Lars Pedersen \\ Henrik Toft Sørensen}

Department of Clinical Epidemiology, Institute of Clinical Medicine, Aarhus University Hospital, Aarhus, Denmark

Correspondence: Sigrun Alba Johannesdottir

Department of Clinical Epidemiology,

Aarhus University Hospital,

Olof Palmes Allé 43-45,

8200 Aarhus N, Denmark

Tel +45 23695947

Fax +45 87I 672I5

Emailsaj@dce.au.dk

\begin{abstract}
The Danish health care system provides partial reimbursement of most prescription medications in Denmark. The dispensation of prescription medications is registered in administrative databases. Each time a prescription is redeemed at a pharmacy, an electronic record is generated with information related to the user, prescriber, the pharmacy, and the dispensed drug. The National Health Service gathers this information for administration of the drug reimbursement plan. Recently, this information became the basis for the establishment of a new research database, the Danish National Database of Reimbursed Prescriptions (DNDRP). In this paper, we review the content, coverage, quality, linkage, access, and research possibilities of this new database. The database encompasses the reimbursement records of all reimbursed drugs sold in community pharmacies and hospital-based outpatient pharmacies in Denmark since 2004 On average, approximately 3.5 million users are recorded in the database each year. During the coverage period, the number of annual prescription redemptions increased by $15 \%$. Most dispensed prescriptions are in the categories "alimentary tract and metabolism", "cardiovascular system", "nervous system", and "respiratory system". Individuals are identified by the unique central personal registration (CPR) number assigned to all persons born in or immigrating to Denmark. The new database fully complies with Denmark's Act on Processing of Personal Data, while avoiding additional restrictions imposed on data use at the Danish National Prescription Registry, administered by Statistics Denmark. Most importantly, CPR numbers are reversibly encrypted, which allows re-identification of drug users; furthermore, the data access is possible outside the servers of Statistics Denmark. These features open additional opportunities for international collaboration, validation studies, studies on adverse drug effects requiring review of medical records, studies involving contact to general practitioners, and linkage of prescription data to other clinical and research databases. The DNDRP thus is a valuable data source for pharmacoepidemiological research.
\end{abstract}

Keywords: Denmark, patient registration, pharmacoepidemiology, registry-based research

\section{Introduction}

Worldwide, prescription registries are the most frequently used data sources in pharmacoepidemiological studies. ${ }^{1-7}$ In particular, the Scandinavian countries Denmark, Norway, and Sweden - have taken a prominent position. ${ }^{2}$ In each of the three countries, all residents are assigned a unique personal identifier, which is used to register social and health services, including prescription dispensations, in various nationwide registries. ${ }^{2,8}$ In Denmark it is the central personal registration [CPR] number. Routine registration of dispensed prescription medications started in Denmark in 1994 , followed by Norway in 2004, and Sweden in $2005 .^{2}$ Other Nordic countries 
have similar registries. ${ }^{2}$ This practice of longitudinal routine registration enables continuous surveillance of the entire Scandinavian population and creates a unique opportunity for registry-based pharmacoepidemiological research with relatively low time and money costs.

Despite this unique research capacity, conduct of large collaborative Nordic or European studies has been hampered by restrictions on access to the data in the Danish National Prescription Registry, accessible through the servers of Statistics Denmark. ${ }^{2,9,10}$ Restrictions placed by Statistics Denmark on data use go beyond the data protection procedures required by the Danish law known as the Act on Processing of Personal Data. ${ }^{11}$ Specifically, Statistics Denmark irreversibly encrypts personal identifiers, allows data access only through its own servers, and restricts statistical output to aggregate tables. These policies preclude studies requiring re-identification of patients for validation studies involving medical record review or contact to general practitioners and patients; furthermore, studies requiring individual pooling of data from other countries, although possible, become cumbersome and time consuming due to the data use restrictions.

A recently established prescription database, titled the Danish National Database of Reimbursed Prescriptions (DNDRP), helps address these limitations. In this paper, we review the data flow, the contents, and research possibilities of this new database.

\section{The Danish health care system}

\section{Structure and organization}

Denmark has a tax-supported welfare system, which guarantees the entire population (5.6 million) health care, education, and social services free at the point of delivery. ${ }^{12}$ Hence, Denmark represents a homogenous population with regard to basic education and access to health care.

The health care system is organized into three politicaladministrative levels: (1) the state, (2) the five regions, and (3) the 98 municipalities. ${ }^{12}$ The state is responsible for legislation, surveillance, national guidelines, and health financing through the Ministry of Health and Prevention. ${ }^{12}$ The five regions are responsible for the delivery of primary and hospital care. General practitioners serve as gatekeepers to health care utilization by providing referrals to hospitals and specialists. ${ }^{12}$ In accordance, the primary health care sector is also responsible for the vast majority of medication prescribing. ${ }^{12,13}$ In 2010, the primary health care sector accounted for $97 \%$ of the total volume of medicinal products sales. ${ }^{13}$ The municipalities are responsible for home nursing, public health, school health, child dental treatment, prevention, and rehabilitation. ${ }^{12}$
Tax revenue finances approximately $85 \%$ of the health care expenses, while the remaining part, which mainly consists of medication and dental costs, is patients' out-ofpocket expenditure. ${ }^{12}$

\section{The drug reimbursement plan}

The Danish health care scheme includes a drug reimbursement plan. ${ }^{12,14}$ Reimbursement is independent of income and can be divided into general reimbursement and individual reimbursement. ${ }^{14}$ The general reimbursement scheme covers most prescription medications in Denmark and can be applied for by the pharmaceutical company at the time of marketing. All residents automatically receive reimbursement for medications covered by general reimbursement, although in some cases fulfillment of additional criteria (eg, specific indications for use) is required. ${ }^{14}$ Such conditional reimbursement may also be granted for selected over-the-counter medications. ${ }^{14}$

Physicians can apply for individual reimbursement on behalf of their patients. ${ }^{14}$ There are four types of individual reimbursement schemes: (1) single reimbursement, (2) increased reimbursement, (3) reimbursement for the chronically ill patient, and (4) reimbursement for the terminally ill patient. ${ }^{14}$ Single reimbursement applies to cases in which an individual may receive reimbursement for one particular drug that he/she requires treatment with and that is not covered under the general reimbursement. ${ }^{14}$ Increased reimbursement can be granted if there is a justified reason for choosing a specific agent over the cheapest medication within the same generic group. ${ }^{14}$ Reimbursement for the chronically ill patient guarantees patients with particularly high annual expenses an annual ceiling of out-of-pocket expenditure (in 2012, the ceiling was 3655 Danish kroner, which currently corresponds to approximately 500 euro or 650 US dollars). ${ }^{14}$ Finally, reimbursement for the terminally ill assures that dying patients who choose to spend their final days outside a hospital are exempted from any medical expenses. ${ }^{14}$

In general, the proportion of reimbursement depends on the patient's age and annual expenses for reimbursable medications. ${ }^{14}$ For children and adolescents younger than 18 years, there is no expenditure threshold for receiving reimbursement. Reimbursement for this age group starts at $60 \%$ and increases with increasing expenditure. Adults become eligible for a $50 \%$ reimbursement after exceeding a certain limit in annual expenses (890 Danish kroner in 2012, which currently corresponds to approximately 120 euro or 150 US dollars), increasing thereafter. Calculations of reimbursement are always based on the cheapest medication 
within each generic group..$^{14}$ If the patient or patient's physician chooses a more expensive medication, he/she must pay the difference or apply for a single reimbursement. ${ }^{14}$

In Denmark, drugs ineligible for general reimbursement currently include, oral contraceptives and benzodiazepines. ${ }^{2,15}$ Information and updates on the reimbursement policies are available at the official website of the Danish Health and Medicines Authority. ${ }^{16}$

\section{Administration of reimbursement}

Danish Regions, is the name of the organization responsible for administering the drug reimbursement plan for the five Danish regions. ${ }^{12}$ Each time a prescription is dispensed, relevant information is recorded directly into the computerized pharmacy system through bar code scanning of the product package. ${ }^{9,15}$ The bar code contains the product number, also termed the Nordic article number. ${ }^{9} 15$ This number is a unique drug identifier, which encodes information on name, form, strength, and pack size of the product. ${ }^{9}{ }^{15}$ Data related to the patient, prescriber, and pharmacy, are also captured..$^{9,15}$ The pharmacy data are then transferred to the responsible region for administration of the drug reimbursement plan. Further data handling results in incorporation of variables such as pharmaceutical form, trade name, Anatomical Therapeutic Chemical (ATC) Classification System code, and Defined Daily Dose (DDD) of the medicinal product. ${ }^{9,17}$

\section{Content and coverage}

Since 2004, the prescription data collected by the Danish Regions have been gathered into a database, which is maintained by the IT-consulting firm Computer Science Corporation (CSC). In March 2011, a contract between Danish Regions and Aarhus University was signed approving the use of the data for research. Thus, the DNDRP was created, with the Department of Clinical Epidemiology at Aarhus University Hospital responsible for database management, financing, and data protection (see 'Data access', below, for further information).

The DNDRP includes data on all reimbursed prescriptions redeemed at Danish community pharmacies and hospitalbased outpatient pharmacies since 2004. It covers the entire Danish population including residents of long-term care institutions. ${ }^{2}$ Since January 1, 1996, information regarding prescriptions redeemed by persons younger than 18 years old should be entered with their own CPR numbers and not that of a parent, as was the case previously. ${ }^{14}$

Because the data are collected with the purpose of reimbursement, data prescriptions dispensed for drugs not receiving reimbursement do not generate records in the DNDRP. In addition, the database contains no data on drugs dispensed during inpatient hospital stays, drugs dispensed directly to patients at outpatient clinics (eg, antiretroviral drugs for HIV treatment or methadone from addiction clinics), over-the-counter drugs (unless approved for reimbursement), and vaccines administered by general practitioners (vaccines are registered in the Danish Vaccination Register). ${ }^{2,15,18}$

Table 1 lists the main variables included in the DNDRP. Of note, CPR numbers are reversibly encrypted in the database.

Tables 2 and 3 show the frequency of users and redeemed prescriptions overall and according to anatomical main groups of the ATC system each year in the DNDRP.

On average, approximately 3.5 million users (defined as persons with one or more prescriptions in a given ATC group) were recorded each year in the database (Table 2). Approximately $48 \%$ of the users each year had redeemed a prescription for "Anti-infectives for systemic use" at least once. Drugs for the "Cardiovascular system" were taken by approximately $30 \%$, "Nervous system" by $27 \%$, "Musculo-skeletal system" by $26 \%$, "Respiratory system" by $23 \%$, and "Alimentary tract and metabolism" and "Dermatologicals" by

Table I List of variables included in the Danish National Database of Reimbursed Prescriptions

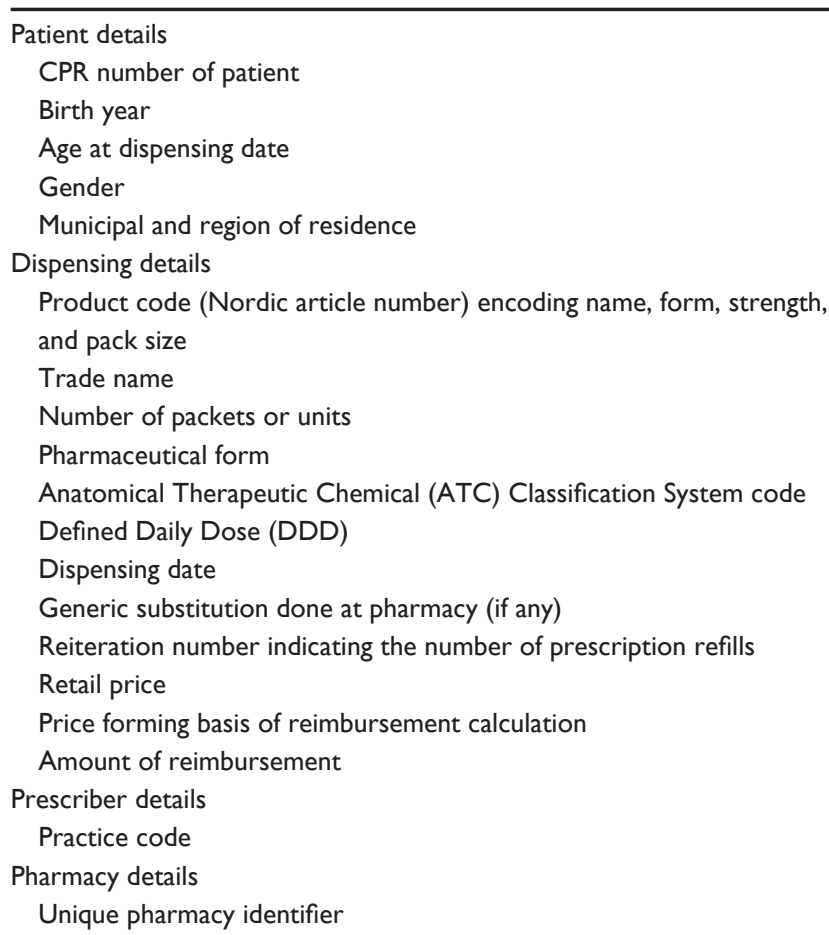

Abbreviations: CPR, central personal registration; ATC, anatomical therapeutic chemical; DDD, defined daily dose. 
Table 2 Distribution of users recorded in the Danish National Database of Reimbursed Prescriptions overall and according to the ATC main groups

\begin{tabular}{|c|c|c|c|c|c|c|c|c|}
\hline \multirow{2}{*}{$\begin{array}{l}\text { ATC anatomical main group, } \\
\text { alphabetically }\end{array}$} & \multicolumn{8}{|c|}{ Number (percent) } \\
\hline & 2004 & 2005 & 2006 & 2007 & 2008 & 2009 & 2010 & 2011 \\
\hline \multicolumn{9}{|l|}{ A } \\
\hline Alimentary tract and metabolism & $\begin{array}{l}624,610 \\
(18.1)\end{array}$ & $\begin{array}{l}644,854 \\
(18.5)\end{array}$ & $\begin{array}{l}677,714 \\
(19.2)\end{array}$ & $\begin{array}{l}704,363 \\
(19.6)\end{array}$ & $\begin{array}{l}734,212 \\
(20.5)\end{array}$ & $\begin{array}{l}753,400 \\
(21.0)\end{array}$ & $\begin{array}{l}801,716 \\
(22.0)\end{array}$ & $\begin{array}{l}820,348 \\
(22.3)\end{array}$ \\
\hline \multicolumn{9}{|l|}{ B } \\
\hline Blood and blood forming organs & $\begin{array}{l}380,650 \\
(I I .0)\end{array}$ & $\begin{array}{l}407,173 \\
(11.7)\end{array}$ & $\begin{array}{l}460,373 \\
(13.0)\end{array}$ & $\begin{array}{l}482,776 \\
(13.5)\end{array}$ & $\begin{array}{l}509,960 \\
(14.2)\end{array}$ & $\begin{array}{l}523,472 \\
(14.6)\end{array}$ & $\begin{array}{l}538,776 \\
(14.8)\end{array}$ & $\begin{array}{l}552,340 \\
(15.0)\end{array}$ \\
\hline \multicolumn{9}{|l|}{ C } \\
\hline Cardiovascular system & $\begin{array}{l}930,340 \\
(26.9)\end{array}$ & $\begin{array}{l}971,670 \\
(27.9)\end{array}$ & $\begin{array}{l}1,028,662 \\
(29.1)\end{array}$ & $\begin{array}{l}I, 082,85 I \\
(30.2)\end{array}$ & $\begin{array}{l}1,135,512 \\
(31.6)\end{array}$ & $\begin{array}{l}1,162,765 \\
(32.3)\end{array}$ & $\begin{array}{l}1,190,876 \\
(32.6)\end{array}$ & $\begin{array}{l}\mathrm{I}, 208,445 \\
(32.8)\end{array}$ \\
\hline \multicolumn{9}{|l|}{$\mathrm{D}$} \\
\hline Dermatologicals & $\begin{array}{l}685,273 \\
(19.8)\end{array}$ & $\begin{array}{l}674,093 \\
(19.4)\end{array}$ & $\begin{array}{l}680,391 \\
(19.2)\end{array}$ & $\begin{array}{l}676,876 \\
(18.9)\end{array}$ & $\begin{array}{l}699,611 \\
(19.5)\end{array}$ & $\begin{array}{l}703,099 \\
(19.6)\end{array}$ & $\begin{array}{l}702,508 \\
(19.2)\end{array}$ & $\begin{array}{l}700,754 \\
(19.0)\end{array}$ \\
\hline \multicolumn{9}{|l|}{ G } \\
\hline $\begin{array}{l}\text { Genito-urinary system and sex } \\
\text { hormones }\end{array}$ & $\begin{array}{l}355,057 \\
(10.3)\end{array}$ & $\begin{array}{l}349,379 \\
(10.0)\end{array}$ & $\begin{array}{l}356,093 \\
(10.1)\end{array}$ & $\begin{array}{l}358,346 \\
(10.0)\end{array}$ & $\begin{array}{l}359,062 \\
(10.0)\end{array}$ & $\begin{array}{l}356,453 \\
(9.9)\end{array}$ & $\begin{array}{l}359,968 \\
(9.9)\end{array}$ & $\begin{array}{l}361,112 \\
(9.8)\end{array}$ \\
\hline \multicolumn{9}{|l|}{$\mathrm{H}$} \\
\hline $\begin{array}{l}\text { Systemic hormonal preparations, } \\
\text { excluding sex hormones and insulins }\end{array}$ & $\begin{array}{l}291,487 \\
(8.4)\end{array}$ & $\begin{array}{l}297,569 \\
(8.5)\end{array}$ & $\begin{array}{l}306,419 \\
(8.7)\end{array}$ & $\begin{array}{l}313,924 \\
(8.7)\end{array}$ & $\begin{array}{l}317,810 \\
(8.9)\end{array}$ & $\begin{array}{l}323,263 \\
(9.0)\end{array}$ & $\begin{array}{l}324,959 \\
(8.9)\end{array}$ & $\begin{array}{l}325,809 \\
(8.8)\end{array}$ \\
\hline \multicolumn{9}{|l|}{ J } \\
\hline Anti-infectives for systemic use & $\begin{array}{l}1,669,319 \\
(48.3)\end{array}$ & $\begin{array}{l}1,699,680 \\
(48.8)\end{array}$ & $\begin{array}{l}1,717,157 \\
(48.6)\end{array}$ & $\begin{array}{l}1,776,125 \\
(49.5)\end{array}$ & $\begin{array}{l}I, 7|8,3| I \\
(47.9)\end{array}$ & $\begin{array}{l}1,703,302 \\
(47.4)\end{array}$ & $\begin{array}{l}I, 771,116 \\
(48.5)\end{array}$ & $\begin{array}{l}I, 812,957 \\
(49.2)\end{array}$ \\
\hline \multicolumn{9}{|l|}{ L } \\
\hline $\begin{array}{l}\text { Antineoplastic and } \\
\text { immunomodulating agents }\end{array}$ & $\begin{array}{l}29,810 \\
(0.9)\end{array}$ & $\begin{array}{l}31,915 \\
(0.9)\end{array}$ & $\begin{array}{l}33,530 \\
(0.9)\end{array}$ & $\begin{array}{l}35,388 \\
(1.0)\end{array}$ & $\begin{array}{l}36,516 \\
(1.0)\end{array}$ & $\begin{array}{l}38,167 \\
(1.1)\end{array}$ & $\begin{array}{l}40,205 \\
(1.1)\end{array}$ & $\begin{array}{l}4 I, 015 \\
(1.1)\end{array}$ \\
\hline \multicolumn{9}{|l|}{ M } \\
\hline Musculo-skeletal system & $\begin{array}{l}972,712 \\
(28.1)\end{array}$ & $\begin{array}{l}968,118 \\
(27.8)\end{array}$ & $\begin{array}{l}961,829 \\
(27.2)\end{array}$ & $\begin{array}{l}948,137 \\
(26.4)\end{array}$ & $\begin{array}{l}946,625 \\
(26.4)\end{array}$ & $\begin{array}{l}923,983 \\
(25.7)\end{array}$ & $\begin{array}{l}926,934 \\
(25.4)\end{array}$ & $\begin{array}{l}908,079 \\
(24.6)\end{array}$ \\
\hline \multicolumn{9}{|l|}{ N } \\
\hline Nervous system & $\begin{array}{l}901,398 \\
(26.1)\end{array}$ & $\begin{array}{l}924,905 \\
(26.6)\end{array}$ & $\begin{array}{l}949,119 \\
(26.8)\end{array}$ & $\begin{array}{l}973,276 \\
(27.1)\end{array}$ & $\begin{array}{l}993,095 \\
(27.7)\end{array}$ & $\begin{array}{l}I, 0 I 6,300 \\
(28.3)\end{array}$ & $\begin{array}{l}I, 057,397 \\
(29)\end{array}$ & $\begin{array}{l}1,065,246 \\
(28.9)\end{array}$ \\
\hline \multicolumn{9}{|l|}{$P$} \\
\hline $\begin{array}{l}\text { Antiparasitic products, insecticides, } \\
\text { and repellents }\end{array}$ & $\begin{array}{l}119,205 \\
(3.4)\end{array}$ & $\begin{array}{l}121,340 \\
(3.5)\end{array}$ & $\begin{array}{l}122,106 \\
(3.5)\end{array}$ & $\begin{array}{l}125,965 \\
(3.5)\end{array}$ & $\begin{array}{l}128,729 \\
(3.6)\end{array}$ & $\begin{array}{l}133,078 \\
(3.7)\end{array}$ & $\begin{array}{l}134,767 \\
(3.7)\end{array}$ & $\begin{array}{l}136,087 \\
(3.7)\end{array}$ \\
\hline \multicolumn{9}{|l|}{$\mathrm{R}$} \\
\hline Respiratory system & $\begin{array}{l}805,083 \\
(23.3)\end{array}$ & $\begin{array}{l}795,370 \\
(22.8)\end{array}$ & $\begin{array}{l}809,077 \\
(22.9)\end{array}$ & $\begin{array}{l}827,|7| \\
(23.0)\end{array}$ & $\begin{array}{l}817,175 \\
(22.8)\end{array}$ & $\begin{array}{l}824,144 \\
(22.9)\end{array}$ & $\begin{array}{l}831,643 \\
(22.8)\end{array}$ & $\begin{array}{l}848,127 \\
(23.0)\end{array}$ \\
\hline \multicolumn{9}{|l|}{ S } \\
\hline Sensory organs & $\begin{array}{l}391,293 \\
(11.3)\end{array}$ & $\begin{array}{l}388,319 \\
(11.2)\end{array}$ & $\begin{array}{l}401,588 \\
(I I .4)\end{array}$ & $\begin{array}{l}402,555 \\
(11.2)\end{array}$ & $\begin{array}{l}401,608 \\
(11.2)\end{array}$ & $\begin{array}{l}405,527 \\
(11.3)\end{array}$ & $\begin{array}{l}397,916 \\
(10.9)\end{array}$ & $\begin{array}{l}399,542 \\
(10.8)\end{array}$ \\
\hline \multicolumn{9}{|l|}{ V } \\
\hline Various & $\begin{array}{l}10,835 \\
(0.3)\end{array}$ & $\begin{array}{l}10,197 \\
(0.3)\end{array}$ & $\begin{array}{l}9,928 \\
(0.3)\end{array}$ & $\begin{array}{l}10,143 \\
(0.3)\end{array}$ & $\begin{array}{l}10,350 \\
(0.3)\end{array}$ & $\begin{array}{l}10,908 \\
(0.3)\end{array}$ & $\begin{array}{l}11,023 \\
(0.3)\end{array}$ & $\begin{array}{l}11,410 \\
(0.3)\end{array}$ \\
\hline Total & $\begin{array}{l}3,456,640 \\
(100.0)\end{array}$ & $\begin{array}{l}3,482,083 \\
(100.0)\end{array}$ & $\begin{array}{l}3,535,169 \\
(100.0)\end{array}$ & $\begin{array}{l}3,589,109 \\
(100.0)\end{array}$ & $\begin{array}{l}3,587,753 \\
(100.0)\end{array}$ & $\begin{array}{l}3,595,303 \\
(100.0)\end{array}$ & $\begin{array}{l}3,651,873 \\
(100.0)\end{array}$ & $\begin{array}{l}3,685,520 \\
(100.0)\end{array}$ \\
\hline Mean number of prescriptions per user & 9.8 & 9.9 & 10.1 & 10.3 & 10.5 & 10.6 & 10.6 & 10.6 \\
\hline
\end{tabular}

Notes: A user is defined as a person with at least one prescription in a given group. Percentages do not add up to $100 \%$ since a person may use more than one category. Abbreviation: ATC, anatomical therapeutic chemical.

approximately $20 \%$ of users each (Table 2 ). The mean number of prescriptions per user was approximately 10 (Table 2).

During the coverage period of the database, the number of prescriptions redeemed per year increased by $15 \%$ (from $33,904,844$ in 2004 to $39,028,817$ in 2011; Table 3 ). The vast majority of prescriptions was accounted for by drugs for the
"Cardiovascular system" (22\%-24\%), "Nervous system" (21\%), "Respiratory system" (10\%-12\%), "Alimentary tract and metabolism" (10\%-11\%), "Anti-infectives for systemic use" (9\%), and drugs for "Musculo-skeletal system" (7\%). This distribution reflects the population burden of chronic diseases treated with these medications, ie, cardiovascular 
Table 3 Distribution of redeemed prescriptions recorded in the Danish National Database of Reimbursed Prescriptions overall and according to the main ATC groups

\begin{tabular}{|c|c|c|c|c|c|c|c|c|}
\hline \multirow{2}{*}{$\begin{array}{l}\text { ATC anatomical main group, } \\
\text { alphabetically }\end{array}$} & \multicolumn{8}{|c|}{ Number (percent) } \\
\hline & 2004 & 2005 & 2006 & 2007 & 2008 & 2009 & 2010 & 2011 \\
\hline \multicolumn{9}{|l|}{ A } \\
\hline Alimentary tract and metabolism & $\begin{array}{l}3,264,402 \\
(9.6)\end{array}$ & $\begin{array}{l}3,381,465 \\
(9.8)\end{array}$ & $\begin{array}{l}3,577,499 \\
(10.0)\end{array}$ & $\begin{array}{l}3,720,123 \\
(10.1)\end{array}$ & $\begin{array}{l}3,909,147 \\
(10.3)\end{array}$ & $\begin{array}{l}3,985,656 \\
(10.5)\end{array}$ & $\begin{array}{l}4,212,712 \\
(10.9)\end{array}$ & $\begin{array}{l}4,293,425 \\
(I I .0)\end{array}$ \\
\hline \multicolumn{9}{|l|}{ B } \\
\hline Blood and blood forming organs & $\begin{array}{l}\mathrm{I}, 463,820 \\
(4.3)\end{array}$ & $\begin{array}{l}I, 586,248 \\
(4.7)\end{array}$ & $\begin{array}{l}I, 794,239 \\
(5.0)\end{array}$ & $\begin{array}{l}\mathrm{I}, 945,108 \\
(5.3)\end{array}$ & $\begin{array}{l}2,081,685 \\
(5.5)\end{array}$ & $\begin{array}{l}2,127,367 \\
(5.6)\end{array}$ & $\begin{array}{l}2,160,083 \\
(5.6)\end{array}$ & $\begin{array}{l}2,209,637 \\
(5.7)\end{array}$ \\
\hline \multicolumn{9}{|l|}{ C } \\
\hline Cardiovascular system & $\begin{array}{l}7,293,131 \\
(21.5)\end{array}$ & $\begin{array}{l}7,576,818 \\
(21.9)\end{array}$ & $\begin{array}{l}7,993,860 \\
(22.4)\end{array}$ & $\begin{array}{l}8,456,255 \\
(22.9)\end{array}$ & $\begin{array}{l}8,922,462 \\
(23.6)\end{array}$ & $\begin{array}{l}8,987,640 \\
(23.7)\end{array}$ & $\begin{array}{l}9,107,921 \\
(23.5)\end{array}$ & $\begin{array}{l}9,121,336 \\
(23.4)\end{array}$ \\
\hline \multicolumn{9}{|l|}{ D } \\
\hline Dermatologicals & $\begin{array}{l}I, 316,773 \\
(3.9)\end{array}$ & $\begin{array}{l}1,296,485 \\
(3.7)\end{array}$ & $\begin{array}{l}1,292,182 \\
(3.6)\end{array}$ & $\begin{array}{l}I, 287,16 \mathrm{I} \\
(3.5)\end{array}$ & $\begin{array}{l}1,309,937 \\
(3.5)\end{array}$ & $\begin{array}{l}\mathrm{I}, 3 \mid 5,287 \\
(3.5)\end{array}$ & $\begin{array}{l}I, 311,512 \\
(3.4)\end{array}$ & $\begin{array}{l}I, 306,42 \mid \\
(3.3)\end{array}$ \\
\hline \multicolumn{9}{|l|}{ G } \\
\hline $\begin{array}{l}\text { Genito-urinary system and sex } \\
\text { hormones }\end{array}$ & $\begin{array}{l}1,223,354 \\
(3.6)\end{array}$ & $\begin{array}{l}1,241,568 \\
(3.6)\end{array}$ & $\begin{array}{l}1,27|,| 3 \mid \\
(3.6)\end{array}$ & $\begin{array}{l}1,281,853 \\
(3.5)\end{array}$ & $\begin{array}{l}1,286,490 \\
(3.4)\end{array}$ & $\begin{array}{l}1,289,222 \\
(3.4)\end{array}$ & $\begin{array}{l}1,316,104 \\
(3.4)\end{array}$ & $\begin{array}{l}1,323,177 \\
(3.4)\end{array}$ \\
\hline \multicolumn{9}{|l|}{$\mathrm{H}$} \\
\hline $\begin{array}{l}\text { Systemic hormonal preparations, } \\
\text { excluding sex hormones and insulins }\end{array}$ & $\begin{array}{l}893,469 \\
(2.6)\end{array}$ & $\begin{array}{l}906,641 \\
(2.6)\end{array}$ & $\begin{array}{l}924,760 \\
(2.6)\end{array}$ & $\begin{array}{l}950,011 \\
(2.6)\end{array}$ & $\begin{array}{l}963,552 \\
(2.6)\end{array}$ & $\begin{array}{l}972,652 \\
(2.6)\end{array}$ & $\begin{array}{l}1,008,675 \\
(2.6)\end{array}$ & $\begin{array}{l}1,026,215 \\
(2.6)\end{array}$ \\
\hline \multicolumn{9}{|l|}{ J } \\
\hline Anti-infectives for systemic use & $\begin{array}{l}3,183,744 \\
(9.4)\end{array}$ & $\begin{array}{l}3,255,075 \\
(9.4)\end{array}$ & $\begin{array}{l}3,312,219 \\
(9.3)\end{array}$ & $\begin{array}{l}3,464,812 \\
(9.4)\end{array}$ & $\begin{array}{l}3,350,631 \\
(8.9)\end{array}$ & $\begin{array}{l}3,299,963 \\
(8.7)\end{array}$ & $\begin{array}{l}3,478,752 \\
(9.0)\end{array}$ & $\begin{array}{l}3,555,367 \\
(9.1)\end{array}$ \\
\hline \multicolumn{9}{|l|}{ L } \\
\hline $\begin{array}{l}\text { Antineoplastic and } \\
\text { immunomodulating agents }\end{array}$ & $\begin{array}{l}90,676 \\
(0.327)\end{array}$ & $\begin{array}{l}96,115 \\
(0.3)\end{array}$ & $\begin{array}{l}95,911 \\
(0.3)\end{array}$ & $\begin{array}{l}100,068 \\
(0.3)\end{array}$ & $\begin{array}{l}105,008 \\
(0.3)\end{array}$ & $\begin{array}{l}108,334 \\
(0.3)\end{array}$ & $\begin{array}{l}112,697 \\
(0.3)\end{array}$ & $\begin{array}{l}114,726 \\
(0.3)\end{array}$ \\
\hline \multicolumn{9}{|l|}{ M } \\
\hline Musculo-skeletal system & $\begin{array}{l}2,691,890 \\
(7.9)\end{array}$ & $\begin{array}{l}2,648,354 \\
(7.7)\end{array}$ & $\begin{array}{l}2,597,139 \\
(7.3)\end{array}$ & $\begin{array}{l}2,533,903 \\
(6.9)\end{array}$ & $\begin{array}{l}2,519,633 \\
(6.7)\end{array}$ & $\begin{array}{l}2,417,428 \\
(6.4)\end{array}$ & $\begin{array}{l}2,388,127 \\
(6.2)\end{array}$ & $\begin{array}{l}2,310,810 \\
(5.9)\end{array}$ \\
\hline \multicolumn{9}{|l|}{$\mathrm{N}$} \\
\hline Nervous system & $\begin{array}{l}7,016,412 \\
(20.7)\end{array}$ & $\begin{array}{l}7,220,883 \\
(20.9)\end{array}$ & $\begin{array}{l}7,434,875 \\
(20.8)\end{array}$ & $\begin{array}{l}7,720,384 \\
(20.9)\end{array}$ & $\begin{array}{l}7,896,890 \\
(20.9)\end{array}$ & $\begin{array}{l}8,028,249 \\
(21.1)\end{array}$ & $\begin{array}{l}8,284,850 \\
(21.3)\end{array}$ & $\begin{array}{l}8,318,380 \\
(21.3)\end{array}$ \\
\hline \multicolumn{9}{|l|}{$P$} \\
\hline $\begin{array}{l}\text { Antiparasitic products, insecticides, } \\
\text { and repellents }\end{array}$ & $\begin{array}{l}215,043 \\
(0.6)\end{array}$ & $\begin{array}{l}217,506 \\
(0.6)\end{array}$ & $\begin{array}{l}219,681 \\
(0.6)\end{array}$ & $\begin{array}{l}224,767 \\
(0.6)\end{array}$ & $\begin{array}{l}228,519 \\
(0.6)\end{array}$ & $\begin{array}{l}232,616 \\
(0.6)\end{array}$ & $\begin{array}{l}237,285 \\
(0.6)\end{array}$ & $\begin{array}{l}237,647 \\
(0.6)\end{array}$ \\
\hline \multicolumn{9}{|l|}{$\mathrm{R}$} \\
\hline Respiratory system & $\begin{array}{l}3,996,386 \\
(11.8)\end{array}$ & $\begin{array}{l}3,930,538 \\
(11.4)\end{array}$ & $\begin{array}{l}3,97|, 36| \\
(I I .1)\end{array}$ & $\begin{array}{l}3,991,830 \\
(10.8)\end{array}$ & $\begin{array}{l}3,937,406 \\
(10.4)\end{array}$ & $\begin{array}{l}3,933,682 \\
(10.4)\end{array}$ & $\begin{array}{l}3,931,911 \\
(10.1)\end{array}$ & $\begin{array}{l}3,945,161 \\
(10.1)\end{array}$ \\
\hline \multicolumn{9}{|l|}{$S$} \\
\hline Sensory organs & $\begin{array}{l}1,234,012 \\
(3.6)\end{array}$ & $\begin{array}{l}1,219,445 \\
(3.5)\end{array}$ & $\begin{array}{l}1,231,742 \\
(3.4)\end{array}$ & $\begin{array}{l}1,228,566 \\
(3.3)\end{array}$ & $\begin{array}{l}1,244,228 \\
(3.3)\end{array}$ & $\begin{array}{l}I, 250,92 I \\
(3.3)\end{array}$ & $\begin{array}{l}1,233,674 \\
(3.2)\end{array}$ & $\begin{array}{l}\mathrm{I}, 242,082 \\
(3.2)\end{array}$ \\
\hline \multicolumn{9}{|l|}{ V } \\
\hline Various & $\begin{array}{l}21,732 \\
(0.1)\end{array}$ & $\begin{array}{l}20,552 \\
(0.1)\end{array}$ & $\begin{array}{l}20,410 \\
(0.1)\end{array}$ & $\begin{array}{l}21,025 \\
(0.1)\end{array}$ & $\begin{array}{l}21,757 \\
(0.1)\end{array}$ & $\begin{array}{l}22,683 \\
(0.1)\end{array}$ & $\begin{array}{l}22,891 \\
(0.1)\end{array}$ & $\begin{array}{l}24,433 \\
(0.1)\end{array}$ \\
\hline Total & $\begin{array}{l}33,904,844 \\
(100.0)\end{array}$ & $\begin{array}{l}34,597,693 \\
(100.0)\end{array}$ & $\begin{array}{l}35,737,009 \\
(100.0)\end{array}$ & $\begin{array}{l}36,925,866 \\
(100.0)\end{array}$ & $\begin{array}{l}37,777,345 \\
(100.0)\end{array}$ & $\begin{array}{l}37,971,700 \\
(100.0)\end{array}$ & $\begin{array}{l}38,807,194 \\
(100.0)\end{array}$ & $\begin{array}{l}39,028,817 \\
(100.0)\end{array}$ \\
\hline
\end{tabular}

Abbreviation: ATC, anatomical therapeutic chemical.

disease, diabetes, psychiatric diseases, and asthma and chronic obstructive pulmonary disease.

\section{Data quality}

The data quality of all registries depends on the completeness of registration (ie, whether all users and prescriptions are included) and the validity of the recorded information (ie, the extent to which a record of an event corresponds to the event). Based on a yet unpublished analysis of crosstabulating records from the DNDRP, with data from Statistics Denmark, we calculated the completeness as the number of users recorded in the DNDRP divided by the number of users recorded by Statistics Denmark. We found a high degree of completeness for most medication groups (Table 4). 
Table 4 Completeness of the Danish National Database of Reimbursed Prescriptions compared with data from Statistics Denmark, according to main ATC groups, $201 \mathrm{I}$

\begin{tabular}{|c|c|c|c|}
\hline \multirow[t]{2}{*}{ ATC main group, alphabetically } & \multicolumn{2}{|l|}{ Number of users } & \multirow[t]{2}{*}{ Completeness } \\
\hline & Statistics Denmark & $\begin{array}{l}\text { Danish National Database } \\
\text { of Reimbursed Prescriptions }\end{array}$ & \\
\hline \multicolumn{4}{|l|}{ A } \\
\hline Alimentary tract and metabolism & 967,662 & 820,348 & 0.84776 \\
\hline \multicolumn{4}{|l|}{ B } \\
\hline Blood and blood forming organs & 630,769 & 552,340 & 0.87566 \\
\hline \multicolumn{4}{|l|}{$\mathrm{C}$} \\
\hline Cardiovascular system & $\mathrm{I}, 347,540$ & $\mathrm{I}, 208,445$ & 0.89678 \\
\hline \multicolumn{4}{|l|}{$\mathrm{D}$} \\
\hline Dermatologicals & $1,019,743$ & 700,754 & 0.68719 \\
\hline \multicolumn{4}{|l|}{ G } \\
\hline Genito-urinary system and sex hormones & 881,901 & 361,112 & 0.40947 \\
\hline \multicolumn{4}{|l|}{$\mathrm{H}$} \\
\hline $\begin{array}{l}\text { Systemic hormonal preparations, excluding } \\
\text { sex hormones and insulins }\end{array}$ & 331,174 & 325,809 & 0.98380 \\
\hline \multicolumn{4}{|l|}{ J } \\
\hline Anti-infectives for systemic use & $1,914,610$ & $\mathrm{I}, 8 \mathrm{I} 2,957$ & 0.94691 \\
\hline \multicolumn{4}{|l|}{$\mathrm{L}$} \\
\hline Antineoplastic and immunomodulating agents & $4 I, 402$ & $4 I, 015$ & 0.99065 \\
\hline \multicolumn{4}{|l|}{ M } \\
\hline Musculo-skeletal system & 972,270 & 908,079 & 0.93398 \\
\hline \multicolumn{4}{|l|}{$\mathrm{N}$} \\
\hline Nervous system & $1,259,843$ & $1,065,246$ & 0.84554 \\
\hline \multicolumn{4}{|l|}{$P$} \\
\hline Antiparasitic products, insecticides, and repellents & 237,957 & 136,087 & 0.57190 \\
\hline \multicolumn{4}{|l|}{$\mathrm{R}$} \\
\hline Respiratory system & 971,306 & 848,127 & 0.87318 \\
\hline \multicolumn{4}{|l|}{ S } \\
\hline Sensory organs & 784,011 & 399,542 & 0.50961 \\
\hline \multicolumn{4}{|l|}{$\mathrm{v}$} \\
\hline Various & 12,317 & 11,410 & 0.92636 \\
\hline
\end{tabular}

Abbreviation: ATC, anatomical therapeutic chemical.

However, relatively low completeness was observed for "Dermatologicals" (68.7\%), "Genito-urinary system and sex hormones" (40.9\%), "Antiparasitic products, insecticides, and repellents" (57.2\%), and "Sensory organs" (51.0\%). A closer examination of the differences at the second level of the ATC code (the therapeutic subgroup) suggests that lack of completeness for these drug types is largely attributable to their reimbursement status (non-reimbursed medications are included in the data from Statistics Denmark). ${ }^{10}$ For example, the drug class "Genito-urinary system and sex hormones" includes oral contraceptives, which are non-reimbursable. A more detailed version of Table 4 is provided in Appendix Table A1.

No study has examined the validity of the data included in the DNDRP. At the same time, data in the Aarhus University Prescription Database (established in 1989), a smaller regional prescription database in Denmark with the same mechanism of record generation, has been shown to have good correspondence with information reported by general practitioners and self-reports for drugs used for chronic conditions. ${ }^{19}$ Since data from the DNDRP come from a similar source, a comparable completeness is likely. Furthermore, the use of bar code scanning and computerized accounting systems in the Danish pharmacies rules out most data entry errors, ensures data uniformity, and minimizes risk of data loss.

\section{Data linkage}

Through linkage to other national or regional registries, which collectively cover most aspects of health and disease in Denmark, the DNDRP may provide new insights into drug utilization patterns, prescribing habits, and intended and unintended drug effects. Thanks to the CPR number, accurate and unambiguous linkage of DNDRP to all major Danish databases is feasible (Figure 1). For example, hospitalization data can be obtained from the Danish National 


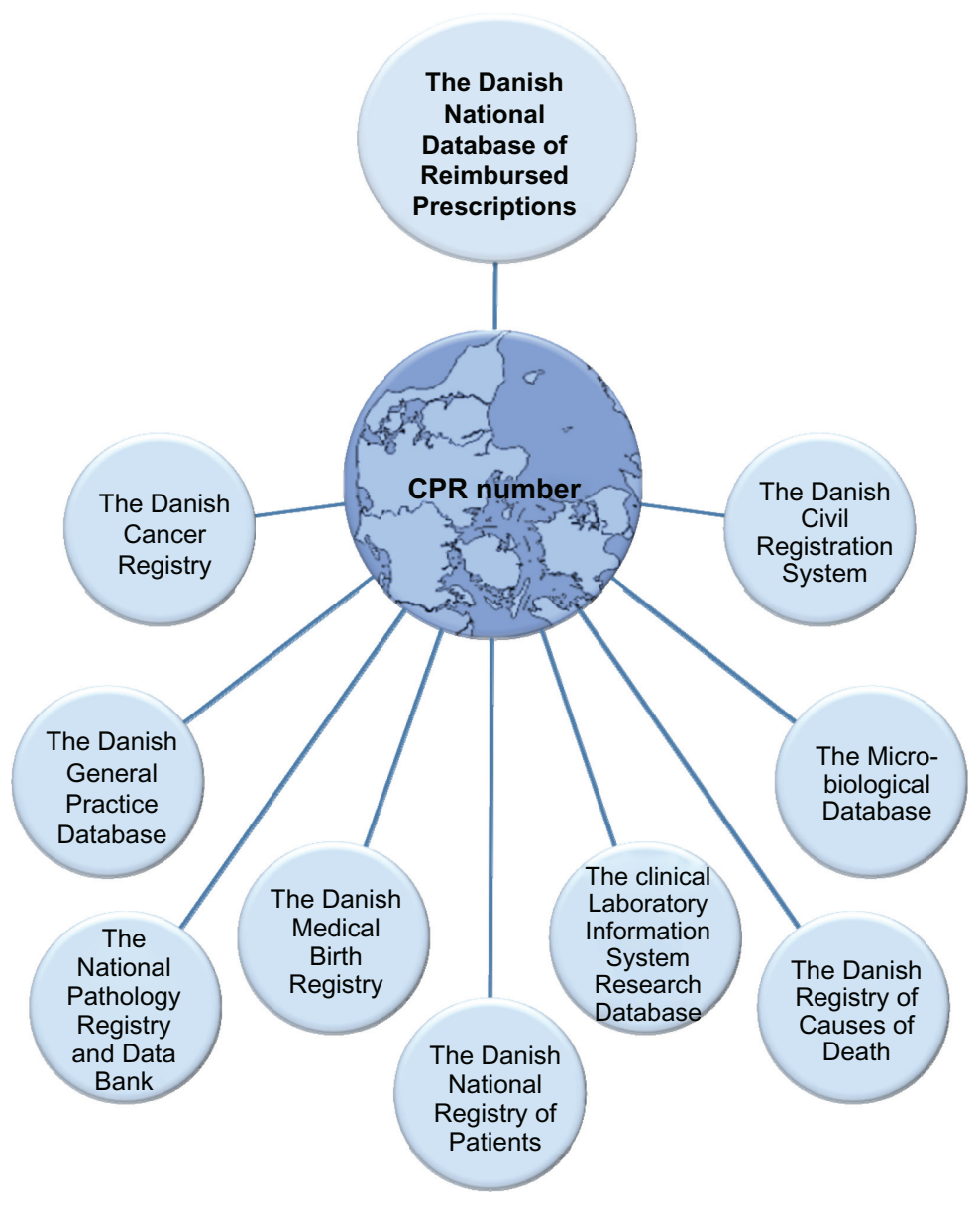

Figure I Selected linkage possibilities of Danish medical databases using the central personal registration (CPR) number. Abbreviation: CPR, central personal registration.

Registry of Patients, which provides information about all inpatient admissions to non-psychiatric hospitals since 1977 and all outpatient clinic and emergency room admissions since $1995 .{ }^{20}$ Daily updates of data on vital status and residence (eg, date of death and emigration) can be retrieved from the Danish Civil Registration System, ${ }^{8}$ while detailed records of all incident malignancies diagnosed in Denmark can be obtained for the Danish Cancer Registry. ${ }^{21}$ Hence, the research possibilities are numerous.

\section{Strengths and limitations}

All of the Nordic countries have nationwide prescription registries containing data similar to that in Denmark. ${ }^{2}$ The prescription registries have several advantages, most importantly that the time spent and costs associated with collection of data are reduced as compared with questionnaire-based studies. Furthermore, the registries have national coverage, and the setting and prospective collection of data limit the likelihood of recall and selection biases. Finally, they include information on dispensed rather than issued prescriptions in contrast to, the General Practice Research Database. ${ }^{3}$ None of the Danish prescription registries contain information on in-hospital medications or non-reimbursable over-the-counter medicine. However, statistics on aggregate sales data for these products are freely accessible at http://www.medstat.dk. ${ }^{22}$

Compared with prescription data at Statistics Denmark, the DNDRP allows for more flexibility because individual records can be analyzed and linked outside the servers of Statistics Denmark. Furthermore, prescriber information is available and CPR numbers are not irreversibly encrypted. These advantages open wider opportunities for more widespread collaboration with other Nordic and European countries, including pooling of individual records, validation studies, studies on adverse drug effects requiring review of medical records, studies involving contact to study subjects' general practitioners and patients, and linkage of prescription data into other clinical databases.

The limitations associated with the DNDRP are similar to those associated with the other prescription databases in Denmark. First, as with all prescription databases, 
there is no information on adherence to prescriptions, which necessitates an assumption of good correspondence between prescription record and drug intake. ${ }^{23}$ Second, indications and instructions for use, prescribed dose, or patient characteristics such as differences in bioavailability, are not included. Hence, in studies of drugs that are not used chronically or with a common regime, assumptions regarding dose and duration of use are required. For some medications, daily dose may be approximated based on the amount dispensed and inter-prescription interval. DDD can also sometimes be used to approximate the prescribed dose. Third, the DNDRP is not suitable for studying nonreimbursed prescription drugs, such as, for example, oral contraceptives. These data are recorded by the Danish National Prescription Registry, which tracks prescriptions regardless of reimbursement status. Fourth, users of the database have to be aware of the fact that ATC codes and DDD may change over time. Finally, the period of coverage only goes back to 2004 . Hence, misclassification may be introduced due to left censoring. Similarly, the length of registration may be inadequate for capturing diseases with a long latent period (eg, cancer) or diagnostic delay (eg, congenital heart diseases). ${ }^{24}$ Although smaller in size, the regional Danish databases (the Aarhus University Prescription Database and the Odense University Pharmacoepidemiologic Database) cover a longer time period, which may be more desirable than a large sample size in certain situations (eg, cancer studies). These needs should therefore be balanced when planning a study.

\section{Perspectives}

Pharmacoepidemiological studies are important for establishing the safety of drugs and the disease burden in the population. Numerous studies using the regional databases have previously been published in high-impact journals, eg, studies on the effects of nonsteroidal anti-inflammatory drugs on the risk of myocardial infarction, ${ }^{25}$ stroke, ${ }^{26}$ venous thromboembolism, ${ }^{27}$ atrial fibrillation or flutter, ${ }^{28}$ cancer, ${ }^{29}$ or adverse birth outcomes or miscarriage. ${ }^{30}$ In addition, the regional databases have been used in drug utilization studies of the pattern of use of lipid-lowering drugs and the use of drug prescriptions for children. ${ }^{31,32}$ These results hold great promise for the DNDRP to become a valuable research tool in the field of clinical epidemiology.

\section{Data access}

The contract between Danish Regions and Aarhus University covers the following five elements:
1. As a collaborative project between Aarhus University and Danish Regions, a research database will be established based on the prescription data collected by the regions.

2. The Department of Clinical Epidemiology will obtain approval for the database establishment by the Data Protection Agency (http://www.datatilsynet.dk/english/) and will ensure that other requirements for data confidentiality are not violated at any point.

3. In accordance with the Act on Processing of Personal Data, ${ }^{11}$ other research groups from the regions may obtain access to the database under the prerequisite that their research protocol has been approved by the chairman of the database, the Data Protection Agency (http:// www.datatilsynet.dk/english/), and, if necessary, by the National Committee on Health Research Ethics (http:// www.dnvk.dk/CVK/home/english.aspx). After seeking information regarding approval by the Data Protection Agency and the National Committee on Health Research Ethics, researchers are therefore welcome to contact the Department of Clinical Epidemiology at Aarhus University Hospital (http://www.kea.au.dk/en/home/).

4. As soon as the database operates, a board will be established with a chairman from the Department of Clinical Epidemiology and members from each of the five regions (Capital Region of Denmark, Region Zealand, Regions of Southern Denmark, Central Denmark Regions, and North Denmark Region) and each of the four Health Science Faculties at the University of Copenhagen, University of Southern Denmark, Aarhus University, and Aalborg University.

5. The Department of Clinical Epidemiology will be in charge of financing all expenses associated with establishment and management of the database.

\section{Conclusion}

The DNDRP contains data on all reimbursed prescription drugs sold in Denmark since 2004. Compared with the Danish National Prescription Registry, maintained at Statistics Denmark, the DNDRP includes prescriber information, CPR numbers are not encrypted, and individual records can be analyzed and linked outside the servers of Statistics Denmark. These advantages expand opportunities for international collaboration; enable validation studies, studies on adverse drug effects requiring review of medical records, studies involving contact to general practitioners, and incorporation of prescription data in other clinical databases. However, information on adherence, indication, or prescribed dose is not included. Furthermore, the coverage period is currently 
relatively short, but more data will accumulate over time. Keeping these weaknesses in mind, the DNDRP provides a valuable tool for pharmacoepidemiological research.

\section{Acknowledgments}

We would like to thank the Department of Clinical Epidemiology Research Foundation and permanent secretary of the ministry of health and previous director at Danish Regions Per Okkels and director at Danish Regions, Lone Christiansen for enabling the establishment of the DNDRP.

\section{Disclosure}

HTS is Chair of the Board for the database. The authors report no conflicts of interest in this work.

\section{References}

1. National Health Inusrance Research Database [database on the Internet]. Taiwan. Available from: http://nhird.nhri.org.tw/en/index.htm. Accessed July 18, 2012.

2. Furu K, Wettermark B, Andersen M, Martikainen JE, Almarsdottir AB, Sørensen HT. The Nordic countries as a cohort for pharmacoepidemiological research. Basic Clin Pharmacol Toxicol. 2010;106(2):86-94.

3. García Rodríguez LA, Pérez Gutthann S. Use of the UK General Practice Research Database for pharmacoepidemiology. Br J Clin Pharmacol. 1998;45(5):419-425.

4. Kozyrskyj AL, Mustard CA. Validation of an electronic, populationbased prescription database. Ann Pharmacother. 1998;32(11) 1152-1157.

5. Ray WA, Griffin MR. Use of Medicaid data for pharmacoepidemiology. Am J Epidemiol. 1989;129(4):837-849.

6. van Herk-Sukel MP, van de Poll-Franse LV, Lemmens VE, et al. New opportunities for drug outcomes research in cancer patients: the linkage of the Eindhoven Cancer Registry and the PHARMO Record Linkage System. Eur J Cancer. 2010;46(2):395-404.

7. Schneeweiss S, Avorn J. A review of uses of health care utilization databases for epidemiologic research on therapeutics. J Clin Epidemiol. 2005;58(4):323-337.

8. Pedersen CB. The Danish civil registration system. Scand J Public Health. 2011;39(Suppl 7):22-25.

9. Danish Health and Medicines Authority [webpage on the Internet] Statistics and analyses. Denmark: Danish Health and Medicines Authority; 2012 [updated June 15, 2012]. Available from: http://laegemiddelstyrelsen. $\mathrm{dk} /$ en/topics/statistics,-prices-and-reimbursement/statistics-andanalyses. Accessed June 13, 2012.

10. Kildemoes HW, Sørensen HT, Hallas J. The Danish National Prescription Registry. Scand J Public Health. 2011;39(Suppl 7):38-41.

11. The Danish Data Protection Agency [webpage on the Internet]. The Act on Processing of Personal Data [in Danish: Persondataloven]. Denmark: The Danish Data Protection Agency; 2007 [updated September 29, 2011]. Available from: http://www.datatilsynet.dk/ lovgivning/persondataloven/. Accessed June 13, 2012.

12. Ministry of Health and Prevention. Health Care in Denmark. Denmark: Ministry of Health and Prevention; 2008. Available from: http://www. sum.dk/Aktuelt/Publikationer/ /media/Filer-Publikationer_i_pdf/2008/ UK_Healthcare_in_dk/pdf.ashx. Accessed June 13, 2012.

13. Danish Health and Medicines Authority [webpage on the Internet]. Drug statistics in Denmark 2006-2010. Denmark: Danish Health and Medicines Authority; 2012 [created August 5, 2011]. Available from: http:// laegemiddelstyrelsen.dk/en/topics/statistics,-prices-and-reimbursement/ statistics-and-analyses/statistics/report-on-the-annual-sale-of-medicines--medstatdk/drug-statistics-2006-2010. Accessed June 13, 2011.
14. Danish Health and Medicines Authority [webpage on the Internet]. Reimbursement. Denmark: Danish Health and Medicines Authority; 2012 [updated April 24, 2012]. Available from: http://laegemiddelstyrelsen.dk/ en/topics/statistics,-prices-and-reimbursement/reimbursement. Accessed June 13, 2012.

15. Ehrenstein V, Antonsen S, Pedersen L. Existing data sources for clinical epidemiology: Aarhus University Prescription Database. Clin Epidemiol. 2010;2:273-279.

16. Official homepage of the Danish Health and Medicines Authority [homepage on the Internet]. Denmark: Danish Health and Medicines Authority; 2012. Available from: http://laegemiddelstyrelsen.dk/en/. Accessed June 13, 2012.

17. WHO Collaborating Centre for Drug Statistics Methodology, Norwegian Institute of Public Health [webpage on the Internet]. ATC/ DDD - History. Norway: WHO Collaborating Centre for Drug Statistics Methodology; 2009 [updated November 19, 2009]. Available from: http://www.whocc.no/atc_ddd_methodology/history/.Accessed June 13, 2012.

18. Grove Krause T, Jakobsen S, Haarh M, Mølbak K. The Danish vaccination register. Euro Surveill. 2012;17(17).

19. Nielsen GL, Sørensen HT, Pedersen AB, Sabroe S. Analyses of data quality in registries concerning diabetes mellitus - a comparison between a population based hospital discharge and an insulin prescription registry. J Med Syst. 1996;20(1):1-10.

20. Lynge E, Sandegaard JL, Rebolj M. The Danish National Patient Register. Scand J Public Health. 2011;39(Suppl 7):30-33.

21. Gjerstorff ML. The Danish Cancer Registry. Scand J Public Health. 2011;39(Suppl 7):42-45.

22. The Danish State Serum Institute [homepage on the Internet]. Statistics on aggregate sales data. Denmark: The Danish State Serum Institute; 2012. Available from http://www.medstat.dk. Accessed June 13, 2012.

23. Johannesdottir SA, Mægbæk ML, Hansen JG, Lash TL, Pedersen L, Ehrenstein V. Correspondence between general practitioner-reported medication use and timing of prescription dispensation. Clin Epidemiol. 2012;4:13-18.

24. Sørensen HT, Sabroe S, Olsen J. A framework for evaluation of secondary data sources for epidemiological research. Int J Epidemiol. 1996;25(22):435-442.

25. Johnsen SP, Larsson H, Tarone RE, et al. Risk of Hospitalization for Myocardial Infarction Among Users of Rofecoxib, Celecoxib, and Other NSAIDs: A Population-Based Case-Control Study. Arch Intern Med. 2005;165(9):978-984.

26. Johnsen SP, Pedersen L, Friis S, et al. Nonaspirin nonsteroidal antiinflammatory drugs and risk of hospitalization for intracerebral hemorrhage: a population-based case-control study. Stroke. 2003;34(2): 387-391.

27. Schmidt M, Christiansen CF, Horváth-Puhó E, Glynn RJ, Rothman KJ, Sørensen HT. Non-steroidal anti-inflammatory drug use and risk of venous thromboembolism. J Thromb Haemost. 2011; 9(7):1326-1333.

28. Schmidt M, Christiansen CF, Mehnert F, Rothman KJ, Sørensen HT. Non-steroidal anti-inflammatory drug use and risk of atrial fibrillation or flutter: population based case-control study. BMJ. 2011;343:d3450.

29. Johannesdottir SA, Chang ET, Mehnert F, Schmidt M, Olesen AB, Sørensen HT. Nonsteroidal anti-inflammatory drugs and the risk of skin cancer: A population-based case-control study. Cancer. 2012; 118(19):4768-4776.

30. Nielsen GL, Sørensen HT, Larsen H, Pedersen L. Risk of adverse birth outcome and miscarriage in pregnant users of non-steroidal anti-inflammatory drugs: population based observational study and case-control study. BMJ. 2001;322(7281):266-270.

31. Riahi S, Fonager K, Toft E, et al. Use of lipid-lowering drugs during 1991-1998 in Northern Jutland, Denmark. Br J Clin Pharmacol. 2001;52(3):307-311.

32. Thrane N, Sørensen HT. A one-year population-based study of drug prescriptions for Danish children. Acta Paediatr. 1999;88(10): $1131-1136$. 


\section{Appendix}

Table A I Completeness of the Danish National Database of Reimbursed Prescriptions compared with data from Statistics Denmark, according to main ATC groups, 2011

\begin{tabular}{|c|c|c|c|}
\hline \multirow[t]{2}{*}{ ATC group, alphabetically } & \multicolumn{2}{|c|}{ Number of users } & \multirow[t]{2}{*}{ Completeness } \\
\hline & $\begin{array}{l}\text { Statistics } \\
\text { Denmark }\end{array}$ & $\begin{array}{l}\text { Danish National Database } \\
\text { of Reimbursed Prescriptions }\end{array}$ & \\
\hline A (alimentary tract and metabolism) & 967,662 & 820,348 & 0.84776 \\
\hline A0I (stomatological preparations) & 79,683 & 32,916 & 0.41309 \\
\hline A02 (drugs for acid related disorders) & 522,244 & 474,602 & 0.90877 \\
\hline A03 (drugs for functional gastrointestinal disorders) & 92,807 & 73,783 & 0.79502 \\
\hline A04 (antiemetics and antinauseants) & 8,623 & 1,595 & 0.18497 \\
\hline A06 (laxatives) & 116,005 & 21,759 & 0.18757 \\
\hline A07 (antidiarrheals, intestinal anti-inflammatory/anti-infective agents) & 76,026 & 58,419 & $0.7684 \mathrm{I}$ \\
\hline A08 (antiobesity preparations, excluding diet products) & 11,135 & 484 & 0.04347 \\
\hline A09 (digestives, including enzymes) & 3,513 & 3,020 & 0.85966 \\
\hline AI0 (drugs used in diabetes) & 213,822 & 210,933 & 0.98649 \\
\hline AlI (vitamins) & 7,563 & 1,870 & 0.24726 \\
\hline AI2 (mineral supplements) & 178,394 & 150,890 & 0.84582 \\
\hline Al4 (anabolic agents for systemic use) & - & - & - \\
\hline AI6 (other alimentary tract and metabolism products) & 36 & 36 & 1.00000 \\
\hline B (blood and blood forming organs) & 630,769 & 552,340 & 0.87566 \\
\hline BOI (antithrombotic agents) & 529,902 & 482,802 & 0.91112 \\
\hline B02 (antihemorrhagics) & $|4,07|$ & 13,858 & 0.98486 \\
\hline B03 (antianemic preparations) & 135,709 & $8 I, 466$ & 0.60030 \\
\hline B05 (blood substitutes and perfusion solutions) & 1,468 & 734 & 0.50000 \\
\hline B06 (other hematological agents) & - & - & - \\
\hline C (cardiovascular system) & $\mathrm{I}, 347,540$ & $\mathrm{I}, 208,445$ & 0.89678 \\
\hline COI (cardiac therapy) & 123,432 & 114,929 & 0.93111 \\
\hline C02 (antihypertensives) & 16,863 & 16,472 & 0.97681 \\
\hline C03 (diuretics) & 498,606 & 480,353 & 0.96339 \\
\hline C04 (peripheral vasodilators) & 218 & 67 & 0.30734 \\
\hline C05 (vasoprotectives) & 162,477 & 1,859 & 0.01144 \\
\hline C07 (beta blocking agents) & 369,616 & 359,072 & $0.97 \mid 47$ \\
\hline C08 (calcium channel blockers) & 386,423 & 374,911 & 0.97021 \\
\hline C09 (agents acting on the renin-angiotensin system) & 663,034 & 637,123 & 0.96092 \\
\hline $\mathrm{ClO}$ (lipid modifying agents) & 599,515 & 583,694 & 0.97361 \\
\hline $\mathrm{D}$ (dermatologicals) & $1,019,743$ & 700,754 & 0.68719 \\
\hline DOI (antifungals for dermatological use) & 328,119 & 164,277 & 0.50066 \\
\hline D02 (emollients and protectives) & 3,610 & 1,930 & 0.53463 \\
\hline D03 (preparations for treatment of wounds and ulcers) & - & - & - \\
\hline D04 (antipruritics, including antihistamines, anesthetics, etc) & 2,470 & 92 & 0.03725 \\
\hline D05 (antipsoriatics) & 34,488 & 31,460 & 0.91220 \\
\hline D06 (antibiotics and chemotherapeutics for dermatological use) & 221,361 & 67,572 & 0.30526 \\
\hline D07 (corticosteroids, dermatological preparations) & 532,753 & 435,394 & 0.81725 \\
\hline D08 (antiseptics and disinfectants) & 100 & I & 0.01000 \\
\hline D09 (medicated dressings) & 54 & 16 & 0.29630 \\
\hline DIO (anti-acne preparations) & 77,020 & 75,847 & 0.98477 \\
\hline DII (other dermatological preparations) & 31,775 & 26,116 & 0.82190 \\
\hline G (genito-urinary system and sex hormones) & 881,901 & 361,112 & 0.40947 \\
\hline G0I (gynecological antiinfectives and antiseptics) & 40,196 & 31,064 & $0.7728 \mathrm{I}$ \\
\hline G02 (other gynecologicals) & 51,872 & 15,564 & 0.30005 \\
\hline G03 (sex hormones and modulators of the genital system) & 665,257 & 221,547 & 0.33302 \\
\hline G04 (urologicals) & $|68| 7 \mid$, & 109,906 & 0.65354 \\
\hline H (systemic hormonal preparations, excluding sex hormones and insulins) & 331,174 & 325,809 & 0.98380 \\
\hline $\mathrm{HOI}$ (pituitary and hypothalamic hormones and analogues) & 20,793 & 20,608 & 0.99110 \\
\hline H02 (corticosteroids for systemic use) & 179,354 & $|77,6| \mid$ & 0.99028 \\
\hline H03 (thyroid therapy) & 138,002 & 134,215 & 0.97256 \\
\hline H04 (pancreatic hormones) & 4,088 & 4,075 & 0.99682 \\
\hline H05 (calcium homeostasis) & 2,290 & 2,227 & 0.97249 \\
\hline
\end{tabular}


Table Al (Continued)

\begin{tabular}{|c|c|c|c|}
\hline \multirow[t]{2}{*}{ ATC group, alphabetically } & \multicolumn{2}{|c|}{ Number of users } & \multirow[t]{2}{*}{ Completeness } \\
\hline & $\begin{array}{l}\text { Statistics } \\
\text { Denmark }\end{array}$ & $\begin{array}{l}\text { Danish National Database } \\
\text { of Reimbursed Prescriptions }\end{array}$ & \\
\hline J (anti-infectives for systemic use) & $1,914,610$ & $1,812,957$ & 0.94691 \\
\hline J0I (antibacterials for systemic use) & $\mathrm{I}, 788,932$ & $\mathrm{I}, 705,15 \mathrm{I}$ & 0.95317 \\
\hline J02 (antimycotics for systemic use) & 144,202 & 143,498 & 0.99512 \\
\hline J04 (antimycobacterials) & 1,053 & $\mathrm{I}, 042$ & 0.98955 \\
\hline J05 (antivirals for systemic use) & 91,266 & 88,303 & 0.96753 \\
\hline J06 (immune sera and immunoglobulins) & 278 & 81 & 0.29137 \\
\hline J07 (vaccines) & 41,004 & $\mathrm{I}, 257$ & 0.03066 \\
\hline L (antineoplastic and immunomodulating agents) & 41,402 & 41,015 & 0.99065 \\
\hline LOI (antineoplastic agents) & 606 & 464 & 0.76568 \\
\hline L02 (endocrine therapy) & 5,533 & 5,445 & 0.98410 \\
\hline L03 (immunostimulants) & 4 & 2 & 0.50000 \\
\hline L04 (immunosuppressants) & 35,434 & 35,272 & 0.99543 \\
\hline M (musculo-skeletal system) & 972,270 & 908,079 & 0.93398 \\
\hline MOI (anti-inflammatory and antirheumatic products) & 847,872 & 829,817 & 0.9787 I \\
\hline M02 (topical products for joint and muscular pain) & $|7,24|$ & 430 & 0.02494 \\
\hline M03 (muscle relaxants) & 47,794 & $11,5 \mid 4$ & 0.24091 \\
\hline M04 (antigout preparations) & 48,135 & 46,710 & 0.97040 \\
\hline M05 (drugs for treatment of bone diseases) & 96,358 & 61,012 & 0.63318 \\
\hline M09 (other drugs for disorders of the musculo-skeletal system) & $\mathrm{I}, 500$ & I,346 & 0.89733 \\
\hline$N$ (nervous system) & $1,259,843$ & $\mathrm{I}, 065,246$ & 0.84554 \\
\hline NOI (anesthetics) & 6,290 & 882 & 0.14022 \\
\hline N02 (analgesics) & 695,944 & 655,803 & 0.94232 \\
\hline N03 (antiepileptics) & 132,667 & II4,548 & 0.86342 \\
\hline N04 (anti-parkinson drugs) & 36,458 & 34,972 & 0.95924 \\
\hline N05 (psycholeptics) & 469,373 & 137,133 & 0.29216 \\
\hline N06 (psychoanaleptics) & 502,320 & 477,523 & 0.95064 \\
\hline N07 (other nervous system drugs) & 70,572 & 33,578 & 0.47580 \\
\hline P (antiparasitic products, insecticides, and repellents) & 237,957 & 136,087 & 0.57190 \\
\hline POI (antiprotozoals) & 163,470 & 135,859 & 0.83109 \\
\hline P02 (antihelminitics) & 71,406 & 200 & 0.00280 \\
\hline P03 (ectoparasiticides, including scabicides, insecticides and repellents) & 5,299 & 45 & 0.00849 \\
\hline $\mathrm{R}$ (respiratory system) & 971,306 & 848,127 & 0.87318 \\
\hline ROI (nasal preparations) & 262,688 & 245,496 & 0.93455 \\
\hline R02 (throat preparations) & 11,197 & 102 & 0.00911 \\
\hline R03 (drugs for obstructive airway diseases) & 456,836 & 454,748 & 0.99543 \\
\hline R05 (cough and cold preparations) & 214,512 & 108,873 & 0.50754 \\
\hline R06 (antihistamines for systemic use) & 274,120 & 232,694 & 0.84888 \\
\hline R07 (other respiratory system products) & - & - & - \\
\hline S (sensory organs) & 784,011 & 399,542 & $0.5096 \mathrm{I}$ \\
\hline SOI (ophthalmological) & 678,838 & 306,007 & 0.45078 \\
\hline SO2 (otologicals) & 80,416 & 42,870 & 0.53310 \\
\hline S03 (ophthalmological and otological preparations) & 68,512 & 68,215 & 0.99566 \\
\hline $\mathrm{V}$ (various) & 12,317 & 11,410 & 0.92636 \\
\hline V0I (allergens) & 10,593 & 10,512 & 0.99235 \\
\hline V03 (all other therapeutic products) & 312 & 80 & 0.25641 \\
\hline V04 (diagnostic agents) & 19 & 4 & 0.21053 \\
\hline V07 (all other non-therapeutic products) & 1,417 & 829 & 0.58504 \\
\hline V08 (contrast media) & - & - & - \\
\hline
\end{tabular}

Abbreviation: ATC, anatomical therapeutic chemical.

Clinical Epidemiology

\section{Publish your work in this journal}

Clinical Epidemiology is an international, peer-reviewed, open access journal focusing on disease and drug epidemiology, identification of risk factors and screening procedures to develop optimal preventative initiatives and programs. Specific topics include: diagnosis, prognosis, treatment, screening, prevention, risk factor modification, systematic

Submit your manuscript here: http://www.dovepress.com/clinical-epidemiology-journal reviews, risk \& safety of medical interventions, epidemiology \& biostatical methods, evaluation of guidelines, translational medicine, health policies \& economic evaluations. The manuscript management system is completely online and includes a very quick and fair peer-review system, which is all easy to use. 